Research Article

\title{
Picard Method for Existence, Uniqueness, and Gauss Hypergeomatric Stability of the Fractional-Order Differential Equations
}

\author{
Zahra Eidinejad $\mathbb{D}^{1},{ }^{1}$ Reza Saadati ${ }^{D},{ }^{1}$ and Manuel De La Sen ${ }^{1}{ }^{2}$ \\ ${ }^{1}$ School of Mathematics, Iran University of Science and Technology, Narmak, Tehran, Iran \\ ${ }^{2}$ Institute of Research and Development of Processes IIDP, University of the Basque Country, Campus of Leioa, Leioa, Spain \\ Correspondence should be addressed to Reza Saadati; rsaadati@eml.cc
}

Received 23 April 2021; Accepted 1 July 2021; Published 21 July 2021

Academic Editor: Zuowei Cai

Copyright (c) 2021 Zahra Eidinejad et al. This is an open access article distributed under the Creative Commons Attribution License, which permits unrestricted use, distribution, and reproduction in any medium, provided the original work is properly cited.

In this paper, we consider a class of fractional-order differential equations and investigate two aspects of these equations. First, we consider the existence of a unique solution, and then, using a new class of control functions, we investigate the Gauss hypergeometric stability. We use Chebyshev and Bielecki norms in order to prove these aspects by the Picard method. Finally, we give some examples to illustrate our results.

\section{Introduction}

The topic of fractional calculus and its significant applications have appeared to be an appropriate tool in study widespread fields of engineering and science. In fact, there are a lot of phenomena in which highly accurate modeling is very important to study them. Fluid mechanics, biological models, electochemistry, viscoelasticity, and electromagnetics are some examples of these fields. Also, research on fractional calculus (FC) and its applications is an important part of mathematical analysis (see [1-6] and references therein). Its importance can also be explored in other fields such as fluid dynamics traffic models, oscillation due to earthquakes, and flow in porous media due to seepage.

Researchers have always tried to create the conditions for the existence and uniqueness of the solution for fractional differential equations. Since most problems cannot be solved for exact solutions, we need powerful analytical techniques. For good results, one needs stable algorithms and methods. For such needs, the stability theory was founded [7, 8]. It has been found that the notion of fractional-order differential equations can well describe these models in science and engendering. Recently, the study of fractional differential equations has been increased among researchers.

Our article is organized as follows. In Section 2, we explain the definitions and some important results that we use in the proofs of this article. In Section 3, we consider the following fractional-order differential equation:

$$
{ }^{c} D_{\mathscr{f}}^{\alpha} \rho(\mathscr{F})=k(\mathscr{F}, \rho(\mathscr{F}), \rho(h(\mathscr{F}))), \quad j \in J \subset \mathbb{R}, \alpha \in(0,1) .
$$

We investigate the existence of a unique solution and the Gauss hypergeometric stability of this fractional-order differential equation.

In the above equation, ${ }^{c} D_{q}^{\alpha}$ for a function $\rho$ given on the interval $J=(0, q], q \in \mathbb{R}_{+}$is the Caputo fractional derivative of order $\alpha$ and the functions $k \in C\left(J \times \mathbb{R}^{2}, \mathbb{R}\right), h \in C(\bar{J}, \bar{J})(\bar{J}$ is the colure of $J$ ) with $h(\mathscr{J}) \leq \mathscr{J}$. In the first theorem, we investigate the Gauss hypergeometric stability of the fractional-order differential equation using the Chebyshev norm, and in the second theorem, we have proved the Gauss hypergeometric stability of the equation by using Bielecki norm. At the end of each theorem, we provide examples that demonstrate our results well. 


\section{Preliminaries}

Discontinuous control strategy is a method that is used in various issues. Among its applications, we can mention the study of the dynamic behavior of the computer worm system (see $[9,10]$, for more information). In this paper, we consider the Gauss hypergeometric function as a control function and use this function to investigate the stability of Gauss hypergeometric. In this section, we present the definitions of the fractional integral, the Riemann-Liouville fractional derivative, and the Caputo fractional derivative of $\operatorname{order} \tau$, which we utilize in this paper, for more detail, we refer to [11-14]. In the continuation, by introducing the Gauss hypergeometric series, we define the Gauss hypergeometric stability of equation (1) [15-17]. Also, we consider the Picard operator and Henry-Gronwall inequality, which we use in the next section $[18,19]$.

Definition 1. Let $\tau>0$ and $k \in C\left(J \times \mathbb{R}^{2}, \mathbb{R}\right)$, and $\Gamma(\tau)$ is the Gamma function. On an interval $[0, \mathscr{J}]$, the Riemann-Liouville fractional integral of order $\tau$ is defined by

$$
I_{\mathscr{J}}^{\tau} k(\mathscr{J})=\frac{1}{\Gamma(\tau)} \int_{0}^{\mathscr{J}} \frac{k(\ell)}{(\mathscr{J}-\ell)^{1-\tau}} \mathrm{d} \ell, \quad \mathscr{J}>0, \tau>0 .
$$

Definition 2. Assume that $n-1<\tau<n, k:[0, \infty) \longrightarrow \mathbb{R}$. The Riemann-Liouville derivative of order $\tau$ and the Caputo derivative of order $\tau$, respectively, are defined by

$$
\begin{aligned}
& { }^{L} D_{\mathcal{F}}^{\tau} k(\mathscr{J})=\frac{1}{\Gamma(n-\tau)} \frac{\mathrm{d}^{n}}{\mathrm{~d} \mathscr{J}^{n}} \int_{0}^{\mathcal{F}} \frac{k(\ell)}{(\mathscr{J}-\ell)^{\tau}+1-n} \mathrm{~d} \ell, \quad \mathscr{J}>0, \\
& { }^{c} D_{\mathscr{J}}^{\tau} k(\mathscr{J})={ }^{L} D_{\mathcal{F}}^{\tau}\left(k(\mathscr{J})-\sum_{k=0}^{n-1} \frac{\mathscr{J}^{k}}{k !} k^{(k)}(0)\right), \quad \mathscr{J}>0 .
\end{aligned}
$$

Now, we consider the Gauss hypergeometric series (see $[17,20]) F$ by

$$
\begin{aligned}
F(\aleph, \beta ; \wp ; z) & =\sum_{k=0}^{\infty} \frac{(\aleph)_{k}(\beta)_{k}}{(\wp)_{k}} \frac{z^{k}}{k !} \\
& =\frac{\Gamma(\wp)}{\Gamma(\aleph) \Gamma(\beta)} \sum_{k=0}^{\infty} \frac{\Gamma(\aleph+k) \Gamma(\beta+k)}{\Gamma(\wp+k)} \frac{z^{k}}{k !},
\end{aligned}
$$

where in $\aleph, \beta, \wp \in \mathbb{R}^{+}$and $z \in \mathbb{R}$.

Remark 1. We consider fractional-order differential equation (1) that is controlled by Gauss hypergeometric $F$. If $w$ is a differential function, satisfying

$$
\left|{ }^{c} D_{\mathscr{J}}^{\alpha} w(t)-k(\mathscr{J}, w(\mathscr{J}), w(h(\mathscr{J})))\right| \leq \varepsilon F\left(\aleph, \beta ; \wp ; \mathscr{J}^{\alpha}\right),
$$

for $k \in C\left(J \times \mathbb{R}^{2}, \mathbb{R}\right)$ and $\varepsilon>0$, then $w$ also satisfies the following integral inequality:

$$
\begin{aligned}
& \left|w(\mathscr{J})-w(0)-\frac{1}{\Gamma(\alpha)} \int_{0}^{\mathscr{J}}(\mathscr{J}-\ell)^{\alpha-1} k(\ell, w(\ell), w(h(\ell))) \mathrm{d} \ell\right| \\
& \leq \frac{\varepsilon}{\Gamma(\alpha)} \int_{0}^{\mathscr{f}}(\mathscr{J}-\ell)^{\alpha-1} F\left(\aleph, \beta ; \wp, \ell^{\alpha}\right) \mathrm{d} \ell \\
& \leq \frac{\varepsilon}{\Gamma(\alpha)} \int_{0}^{\mathscr{J}}(\mathscr{J}-\ell)^{\alpha-1} \frac{\Gamma(c)}{\Gamma(\aleph) \Gamma(\beta)} \sum_{k=0}^{\infty} \frac{\Gamma(\aleph+k) \Gamma(\beta+k)}{\Gamma(\wp+k)} \frac{\ell^{\alpha k}}{k !} \mathrm{d} \ell \\
& =\frac{\varepsilon}{\Gamma(\alpha)} \frac{\Gamma(\wp)}{\Gamma(\aleph) \Gamma(\beta)} \sum_{k=0}^{\infty} \frac{\Gamma(\aleph+k) \Gamma(\beta+k)}{\Gamma(\wp+k)} \frac{1}{k !} \int_{0}^{\mathscr{f}}(\mathscr{J}-\ell)^{\alpha-1} \ell^{\alpha k} \mathrm{~d} \ell \\
& =\frac{\varepsilon}{\Gamma(\alpha)} \frac{\Gamma(c)}{\Gamma(a) \Gamma(b)} \sum_{k=0}^{\infty} \frac{\Gamma(\aleph+k) \Gamma(\beta+k)}{\Gamma(\wp+k)} \frac{\mathcal{J}^{(k+1) \alpha} \Gamma(k \alpha+1) \Gamma(\alpha)}{\Gamma((k+1) \alpha+1) k !} \\
& \leq \varepsilon \frac{\Gamma(\wp)}{\Gamma(\aleph) \Gamma(\beta)} \sum_{n=0}^{\infty} \frac{\Gamma(\aleph+k) \Gamma(\beta+k)}{\Gamma(\wp+k)} \frac{\mathcal{g}^{n \alpha} \Gamma(n \alpha+1)}{\Gamma((n+1) \alpha+1) n !} \\
& \leq \varepsilon \frac{\Gamma(\wp)}{\Gamma(\aleph) \Gamma(\beta)} \sum_{n=0}^{\infty} \frac{\Gamma(\aleph+k) \Gamma(\beta+k)}{\Gamma(\wp+k)} \frac{1}{n !} \frac{\mathcal{J}^{n \alpha} \Gamma(n \alpha+1)}{\Gamma(n \alpha+1)} \\
& =\varepsilon F\left(\aleph, \beta ; \wp, \mathcal{J}^{\alpha}\right) \text {. }
\end{aligned}
$$

Remark 2. Assume that $w$ is a function such that $w \in C(J, \mathbb{R})$. Then, $w$ is a solution of inequality (5) if and only if we can find a function $\widetilde{f}_{w} \in C(J, \mathbb{R})$ such that (i) $|\tilde{f}(\mathscr{J})| \leq \varepsilon F\left(\aleph, \beta ; \wp ; \mathscr{J}^{\alpha}\right)$, for all $\mathscr{J} \in J$

(ii) ${ }^{c} D_{\mathscr{J}}^{\alpha} w(\mathscr{J})=k(\mathscr{J}, w(\mathscr{J}), w(h(\mathscr{J})))+\widetilde{f}(\mathscr{J})$, for all $\mathscr{J} \in J$ 
Definition 3. We say that equation (1) is Gauss hypergeometric stable w.r.t $F\left(\aleph, \beta ; \wp ; \mathscr{J}^{\alpha}\right)$ if, for each $\varepsilon>0$, there exists a constant value $c_{F}>0$, such that, for a differentiable function $w$ satisfying (5), we can find $\rho$ such that the following holds:

$$
{ }^{c} D_{\mathscr{J}}^{\alpha} \rho(\mathscr{J})=k(\mathscr{J}, \rho(\mathscr{J}), \rho(h(\mathscr{J}))), \quad \mathscr{J} \in J \subset \mathbb{R}, \alpha \in(0,1) .
$$

Then, we have

$$
|w(\mathscr{J})-\rho(\mathscr{J})| \leq c_{F} \varepsilon F\left(\aleph, \beta ; \wp ; \mathscr{J}^{\alpha}\right), \quad \mathscr{J} \in J
$$

Definition 4. Consider the metric space $(Y, \delta)$ and the operator $T$ on $Y$. Now, $T$ is a Picard operator if there exists $y^{*} \in Y$ such that

(i) $E_{T}=y^{*}$ wherever $E_{T}=\{y \in Y: T(y)=y\}$ is the fixed point set of $T$

(ii) The sequence $\left(T^{n}\left(y_{0}\right)\right)_{n \in \mathbb{N}}$ converges to $y^{*}$, for all $y_{0} \in Y$.

Lemma 1. If we consider an increasing Picard operator $T: Y \longrightarrow Y$, then $(Y, \delta, \leq)$ is an ordered metric space. Therefore, for each $y \in Y, y \leq T(y)$, we have $y \leq y_{T}^{*}$.

In the sequel, we express the Gronwall lemma (see Lemma 7.1.1 in [21]).

Lemma 2. We consider the continuous functions $\mu, v: J \longrightarrow[0, \infty)$. For the nondecreasing function $v$, there are positive constants $\kappa$ and $0<\alpha<1$ such that

$$
\mu(\mathscr{J}) \leq \nu(\mathscr{J})+\kappa \int_{0}^{\mathscr{F}}(\mathscr{J}-\ell)^{\alpha-1} \mu(\ell) \mathrm{d} \ell, \quad \mathscr{J} \in J .
$$

Therefore,

$$
\mu(\mathscr{J}) \leq \nu(\mathscr{J})+\int_{0}^{\mathscr{F}}\left(\sum_{n=1}^{\infty} \frac{(\kappa \Gamma(\alpha))^{n}}{\Gamma(n \alpha)}(\mathscr{J}-\ell)^{n \alpha-1} \nu(\ell)\right) \mathrm{d} \ell, \quad \mathscr{J} \in J
$$

Remark 3. For continuous and nondecreasing functions $\nu(j)$ and $\mu(\mathscr{J})$ on $J$, we have

$$
\mu(\mathscr{J}) \leq \nu(\mathscr{J}) F\left(\aleph, \beta ; \wp ; \kappa \Gamma(\alpha) \mathscr{J}^{\alpha}\right)
$$

Remark 4. We propose the Picard method for the uniqueness and stability of this problem, in which case both its conditions will be weaker and it will be easier to prove it.

Remark 5 (see [22]). There are some slight mistakes and typos in [13]. In Definition 2.4 and Remark 2.6 in [13], the space $C[-h, d]$ should be $C[-h, 0] \cap C_{1-\alpha ; \psi}[0, d]$. For the precise definition needed in [13], we note that the statement (some typos) and proof of Theorem 1 in [13] is fine once $X=C[-h, d]$ in $[13]$ is replaced by $C[-h, 0] \cap C_{1-\alpha ; \psi}[0, d]$ with the norm (there was accidently a typo in relation to the norm in [13]) $\max \left\{\|\cdot\| C[-h, 0],\|\cdot\|_{C 1-\alpha ; \psi[0, d]}\right\}$.

\section{Main Results}

As mentioned in Section 1, we use stability algorithms and methods to get good results. As we know, there are different types of stability such as exponential, Mittag-Leffler and Lyapunov type (see $[9,23]$, for more details). Here, we prove the Gauss hypergeometric stability of equation (1). Before proving the Gauss hypergeometric stability, we first investigate the existence of a unique solution to the fractionalorder differential equation. We prove the first theorem utilizing Chebyshev norm $\|\cdot\|_{C}\left(\|\rho\|_{C}=\max _{\mathscr{J} \in J}|\rho(\mathscr{J})|\right)$.

Theorem 1. We assume that the functions $k \in C\left(J \times \mathbb{R}^{2}, \mathbb{R}\right)$ and $h \in C(\bar{J}, \bar{J})$ exist, such that $h(\mathscr{J}) \leq \mathscr{J}$. Assume the following are satisfied:

(i) There exists $\mathbf{L}_{k}>0$ such that $\mid k\left(\mathscr{J}, u_{1}, u_{2}\right)-$ $k\left(\mathscr{J}, v_{1}, v_{2}\right)\left|\leq \mathbf{L}_{k} \sum_{i=1}^{2}\right| u_{i}-v_{i} \mid$, for all $\mathscr{J} \in J, u_{i}, v_{i} \in$ $\mathbb{R}, i=1,2$

(ii) $\left(2 \mathbf{L}_{k} q^{\alpha} / \Gamma(\alpha+1)\right)<1$

Therefore, (1) has a unique solution in $C(J, \mathbb{R})$ and is Gauss hypergeometric stable.

Proof. According to equation (1), we have

$$
\rho(\mathscr{J})=\varphi(0)+\frac{1}{\Gamma(\alpha)} \int_{0}^{\mathscr{J}}(\mathscr{J}-\ell)^{\alpha-1} k(\ell, \rho(\ell), \rho(h(\ell))) \mathrm{d} \ell, \quad \mathscr{J} \in J .
$$

It is easy to see that (1) is equivalent to (12).

We first prove the existence of solution for equation (12). We define the mapping $\Lambda_{k}$ in $Y:=C(J, \mathbb{R})$ as

$$
\Lambda_{k} \rho(\mathscr{J})=\varphi(0)+\frac{1}{\Gamma(\alpha)} \int_{0}^{\mathscr{J}}(\mathscr{J}-\ell)^{\alpha-1} k(\ell, \rho(\ell), \rho(h(\ell))) \mathrm{d} \ell, \quad \mathscr{J} \in J .
$$

Now, we need to demonstrate that $\Lambda_{k}$ determined in (13) is a contraction mapping on $Y$ : $=C(J, \mathbb{R})$. 
According (i), we have

$$
\begin{aligned}
& \left|\Lambda_{k} \rho(\mathscr{J})-\Lambda_{k} w(\mathscr{J})\right| \\
& \leq \frac{1}{\Gamma(\alpha)} \int_{0}^{\mathscr{F}}(\mathscr{J}-\ell)^{\alpha-1}\left|\begin{array}{c}
k(\ell, \rho(\ell), \rho(h(\ell))) \\
-k(\ell, w(\ell), w(h(\ell)))
\end{array}\right| \mathrm{d} \ell \\
& \leq \frac{\mathbf{L}_{k}}{\Gamma(\alpha)} \int_{0}^{\mathscr{J}}(\mathscr{J}-\ell)^{\alpha-1}\left(\max _{0 \leq \ell \leq q}|\rho(\ell)-w(\ell)|+\max _{0 \leq \ell \leq q}|\rho(h(\ell))-w(h(\ell))|\right) \mathrm{d} \ell \\
& \leq \frac{2 \mathbf{L}_{k}}{\Gamma(\alpha)}\|\rho-w\|_{\mathcal{c}} \int_{0}^{\mathscr{F}}(\mathscr{J}-\ell)^{\alpha-1} \mathrm{~d} \ell \\
& \leq \frac{2 \mathbf{L}_{k} q^{\alpha}}{\Gamma(\alpha+1)}\|\rho-w\|_{\mathcal{C}},
\end{aligned}
$$

for all $\mathscr{J} \in J$, which implies that

$$
\left\|\Lambda_{k}(\rho)-\Lambda_{k}(w)\right\|_{C} \leq \frac{2 \mathbf{L}_{k} q^{\alpha}}{\Gamma(\alpha+1)}\|\rho-w\|_{C}, \quad \rho, w \in C(J, \mathbb{R}) .
$$

Thus, $\Lambda_{k}$ is a contraction mapping w.r.t. the Chebyshev norm $\|.\|_{C}$ on $Y$. Now, to continue the proof, we utilize the Banach contraction principle.
Now, we prove Gauss hypergeometric stability. We assume that $\rho$ is a unique solution to equation (1). Then, for $w \in C(J, \mathbb{R})$ that is a given differentiable function, satisfying (5), we have

$$
\rho(\mathscr{J})=w(0)+\frac{1}{\Gamma(\alpha)} \int_{0}^{\mathscr{J}}(\mathscr{J}-\ell)^{\alpha-1} k(\ell, \rho(\ell), \rho(h(\ell))) \mathrm{d} \ell, \quad \mathscr{J} \in J .
$$

Obviously, Remark 1 implies that

$$
\left|w(\mathscr{J})-w(0)-\frac{1}{\Gamma(\alpha)} \int_{0}^{\mathscr{F}}(\mathscr{J}-\ell)^{\alpha-1} k(\ell, w(\ell), w(h(\ell))) \mathrm{d} \ell\right| \leq \varepsilon F\left(\aleph ; \beta ; \wp ; \mathscr{J}^{\alpha}\right),
$$

for $\mathscr{J} \in J$.

Also according to (i), we have

$$
\begin{aligned}
&|w(\mathscr{J})-\rho(\mathscr{J})| \leq\left|w(\mathscr{J})-w(0)-\frac{1}{\Gamma(\alpha)} \int_{0}^{\mathscr{F}}(\mathscr{J}-\ell)^{\alpha-1} k(\ell, w(\ell), w(h(\ell))) \mathrm{d} \ell\right| \\
&+\mid \frac{1}{\Gamma(\alpha)} \int_{0}^{\mathscr{F}}(\mathscr{J}-\ell)^{\alpha-1} k(\ell, w(\ell), w(h(\ell))) \mathrm{d} \ell \\
&-\frac{1}{\Gamma(\alpha)} \int_{0}^{\mathscr{J}}(\mathscr{J}-\ell)^{\alpha-1} k(\ell, \rho(\ell), \rho(h(\ell))) \mathrm{d} \ell \\
& \leq \varepsilon F\left(\aleph, \beta ; \wp ; \mathscr{J}^{\alpha}\right)+\frac{\mathbf{L}_{k}}{\Gamma(\alpha)}\left(\int_{0}^{\mathscr{F}}(\mathscr{J}-\ell)^{\alpha-1}[|w(\ell)-\rho(\ell)| \mathrm{d} \ell+|w(h(\ell))-\rho(h(\ell))|] \mathrm{d} \ell\right),
\end{aligned}
$$

for all $g \in J$. Now, we consider the operator $T: C\left(J, \mathbb{R}_{+}\right) \longrightarrow C\left(J, \mathbb{R}_{+}\right)$, for each $\sigma \in C\left(J, \mathbb{R}_{+}\right)$, as follows:

$$
T \sigma(\mathscr{J})=\varepsilon F\left(\aleph, \beta ; \wp ; \mathscr{J}^{\alpha}\right)+\frac{\mathbf{L}_{k}}{\Gamma(\alpha)}\left(\int_{0}^{\mathscr{J}}(\mathscr{J}-\ell)^{\alpha-1} \sigma(\ell) \mathrm{d} \ell+\int_{0}^{\mathscr{J}}(\mathscr{J}-\ell)^{\alpha-1} \sigma(h(\ell)) \mathrm{d} \ell\right), \quad \mathscr{J} \in J
$$


In the continuation, we show that $T$ is a Picard operator.

By (ii), we have

$$
\begin{aligned}
& |T \sigma(\mathscr{J})-T \vartheta(\mathscr{J})| \\
& \leq \frac{\mathbf{L}_{k}}{\Gamma(\alpha)}\left(\int_{0}^{\mathscr{F}}(\mathscr{J}-\ell)^{\alpha-1}[|\sigma(\ell)-\vartheta(\ell)| \mathrm{d} \ell+|\sigma(h(\ell))-\vartheta(h(\ell))|] \mathrm{d} \ell\right) \\
& \leq \frac{2 \mathbf{L}_{k} q^{\alpha}}{\Gamma(\alpha+1)}\|\sigma-\vartheta\|_{C},
\end{aligned}
$$

for all $\mathscr{J} \in J$ and for every $\sigma, \vartheta \in C\left(J, \mathbb{R}_{+}\right)$, which implies that

$$
\|T(\sigma)-T(\vartheta)\|_{C} \leq \frac{2 \mathbf{L}_{k} q^{\alpha}}{\Gamma(\alpha+1)}\|\sigma-\vartheta\|_{C}
$$

Consequently, $T$ w.r.t. the Chebyshev norm $\|.\|_{C}$ is a contraction mapping on $C\left(J, \mathbb{R}_{+}\right)$. Thus, utilizing the Banach contraction principle, we obtain that $T$ is a Picard operator and $E_{T}=\left\{\sigma^{*}\right\}$. Now, for,

$$
\sigma^{*}(\mathscr{J})=\varepsilon F\left(\aleph, \beta ; \wp ; \mathscr{J}^{\alpha}\right)+\frac{\mathbf{L}_{k}}{\Gamma(\alpha)}\left(\int_{0}^{\mathscr{J}}(\mathscr{J}-\ell)^{\alpha-1} \sigma^{*}(\ell) \mathrm{d} \ell+\int_{0}^{\mathscr{J}}(\mathscr{J}-\ell)^{\alpha-1} \sigma^{*}(h(\ell)) \mathrm{d} \ell\right) .
$$

In the sequel, we show that the solution $\sigma^{*}$ is increasing. Suppose that and denote $m:=\min _{\ell \in J}\left[\sigma^{*}(\ell)+\sigma^{*}(h(\ell))\right]$ $\in \mathbb{R}_{+}$, and we have

$$
\begin{aligned}
\sigma^{*}\left(\mathscr{J}_{2}\right)-\sigma^{*}\left(\mathscr{J}_{1}\right)= & \varepsilon\left[F\left(\aleph, \beta ; \wp ; \mathscr{J}_{2}^{\alpha}\right)-F\left(\aleph, \beta, \wp ; \mathscr{J}_{1}^{\alpha}\right)\right] \\
& \frac{\mathbf{L}_{k}}{\Gamma(\alpha)} \int_{0}^{\mathscr{F}_{1}}\left[\left(\mathscr{J}_{2}-\ell\right)^{\alpha-1}-\left(\mathscr{J}_{1}-\ell\right)^{\alpha-1}\right]\left[\sigma^{*}(\ell)+\sigma^{*}(h(\ell))\right] \mathrm{d} \ell \\
& +\frac{\mathbf{L}_{k}}{\Gamma(\alpha)} \int_{\mathscr{J}_{1}}^{\mathscr{F}_{2}}\left(\mathscr{J}_{2}-\ell\right)^{\alpha-1}\left[\sigma^{*}(\ell)+\sigma^{*}(h(\ell))\right] \mathrm{d} \ell \geq \varepsilon\left[F\left(\aleph, \beta ; \wp ; \mathscr{F}_{2}^{\alpha}\right)-F\left(\aleph, \beta ; \wp ; \mathscr{J}_{1}^{\alpha}\right)\right] \\
& +\frac{m \mathbf{L}_{k}}{\Gamma(\alpha)} \int_{0}^{\mathscr{F}_{1}}\left[\left(\mathscr{J}_{2}-\ell\right)^{\alpha-1}-\left(\mathscr{J}_{1}-\ell\right)^{\alpha-1}\right] \mathrm{d} \ell+\frac{m \mathbf{L}_{k}}{\Gamma(\alpha)} \int_{\mathscr{J}_{1}}^{\mathscr{F}_{2}}\left(\mathscr{J}_{2}-\ell\right)^{\alpha-1} \mathrm{~d} \ell \\
= & \varepsilon\left[F\left(\aleph, \beta ; \wp ; \mathscr{J}_{2}^{\alpha}\right)-F\left(\aleph, \beta ; \wp ; \mathscr{J}_{1}^{\alpha}\right)\right]+\frac{m \mathbf{L}_{k}}{\Gamma(\alpha+1)}\left(\mathscr{F}_{2}^{\alpha}-\mathscr{J}_{1}^{\alpha}\right)>0 .
\end{aligned}
$$

Then, $\sigma^{*}$ is increasing. From $h(\mathscr{J}) \leq \mathscr{J}$, we obtain $\sigma^{*}(h(\mathscr{J})) \leq \sigma^{*}(\mathscr{J})$

$$
\sigma^{*}(\mathscr{J}) \leq \varepsilon F\left(\aleph, \beta ; \wp ; \mathscr{J}^{\alpha}\right)+\frac{2 \mathbf{L}_{k}}{\Gamma(\alpha)} \int_{0}^{\mathscr{J}}(\mathscr{J}-\ell)^{\alpha-1} \sigma^{*}(\ell) \mathrm{d} \ell .
$$

Now, utilizing Lemma 2, Remark 2, and $c_{F}:=F\left(\aleph, \beta ; \wp ; 2 \mathbf{L}_{k} q^{\alpha}\right)$, we obtain

$$
\sigma^{*}(\mathscr{J}) \leq c_{F} \varepsilon F\left(\aleph, \beta ; \wp ; \mathscr{J}^{\alpha}\right), \quad \mathscr{J} \in J .
$$

Since $T$ is a Picard operator and increasing, by Lemma 1 and from (18), we conclude that $\sigma \leq \sigma^{*}$. If we consider $\sigma=|w-\rho|$, then

$$
|w(\mathscr{J})-\rho(\mathscr{J})| \leq c_{F} \varepsilon F\left(\aleph, \beta ; w p ; \mathscr{J}^{\alpha}\right), \quad \mathscr{J} \in J .
$$
stable.

Therefore, equation (1) is Gauss hypergeometric

In the following, we give an example to illustrate the previous theorem. 
Example 1. Consider a differential equation for $\alpha=1 / 2$ and for any $\mathscr{J} \in(0,1](q=1)$ as follows:

$$
{ }^{c} D_{\mathscr{J}}^{1 / 2} \rho(\mathscr{J})=0.01 \rho^{5 / 2} \sqrt{\pi}(1+\mathscr{J})^{-(3 / 2)}, \quad \mathscr{J} \in(0,1] .
$$

Based on what has been said in the above theorem, we have

$$
\rho(\mathscr{J})=w(0)+\frac{0.01}{\Gamma(1 / 2)} \int_{0}^{\mathscr{F}}(\mathscr{J}-\ell)^{-(1 / 2)} \rho(\mathscr{J})^{5 / 2} \sqrt{\pi}(1+\ell)^{-(3 / 2)} \mathrm{d} \ell .
$$

According to equation (5) and using the fractional-order differential equation, the following inequality is established for the differentiable function $w$ :

$$
\left|{ }^{c} D_{\mathscr{J}}^{1 / 2} w(\mathscr{J})-k\left(\mathscr{J}, w(\mathscr{J}), w\left((1+\mathscr{J})^{-(3 / 2)}\right)\right)\right| \leq \varepsilon F\left(\aleph, \beta ; \wp ; \mathscr{J}^{1 / 2}\right) .
$$

The functions $k$ and $h$ used in the above inequality are considered as follows:

$$
\begin{aligned}
h(\mathfrak{I}) & =(1+\mathfrak{I})^{-(3 / 2)} \\
k(\mathfrak{I}, \rho(\mathfrak{I}), \rho(h(\mathfrak{I}))) & =0.01 \rho^{5 / 2} \sqrt{\pi}(1+\mathfrak{I})^{-(3 / 2)} .
\end{aligned}
$$

To be Ulam-Hyers-hypergeometric stable, we need a positive constant $\mathbf{L}_{k}$ and a coefficient of Lipschitz $2 \mathbb{L}_{k} q^{\alpha} / \Gamma(\alpha+1)$. Then, we consider $\mathbf{L}_{k}=2 / 5$ and as a result $2 \mathbb{L}_{k} q^{\alpha} / \Gamma(\alpha+1)=8 / 5 \sqrt{\pi} \approx 0.9<1$.

According to Theorem 1, all the conditions for the existence of the solution and the Gauss hypergeometric stability are established. Thus, the coefficient $c_{F}=F(\aleph$, $\beta ; \wp ;(4 / 5))$ exists to us so that

$$
|w(\mathscr{J})-\rho(\mathscr{J})| \leq c_{F} \varepsilon F\left(\aleph, \beta ; \wp ; \mathscr{J}^{1 / 2}\right), \quad \mathscr{J} \in(0,1] .
$$

Based on equation (28), we have a nonlinear equation whose diagram for the arbitrary values in the interval $(0,1]$ is shown in Figure 1.

In the next theorem, we prove the Gauss hypergeometric stability utilizing Bielecki's norm $\|\cdot\|_{B}\left(\|\rho\|_{B}:=\right.$ $\left.\max _{\mathscr{J} \in I}|\rho(\mathscr{J})| \exp (-\eta \mathscr{J}), \eta>0, I \subset \mathbb{R}_{+}\right)$.

Theorem 2. Assume that the functions $k \in C\left(J \times \mathbb{R}^{2}, \mathbb{R}\right)$, $h \in C(\bar{J}, \bar{J})$ exist, such that $h(\mathscr{J}) \leq \mathscr{J}$. Assume the following are satisfied:

(i) There exists $\mathbf{L}_{k}>0$ such that $\mid k\left(\mathscr{J}, u_{1}, u_{2}\right)-k(\mathscr{J}$, $\left.v_{1}, v_{2}\right)\left|\leq \mathbf{L}_{k} \sum_{i=1}^{2}\right| u_{i}-v_{i} \mid$ for all $\mathscr{J} \in J, u_{i}, \quad v_{i} \in \mathbb{R}$, $i=1,2$

(ii) $\left(2 \mathbf{L}_{k} q^{\alpha} \exp (\eta q) / \Gamma(\alpha) \sqrt{2(2 \alpha-1) \eta}\right)<1$, for some $\alpha \in(1 / 2,1)$ and $\eta>0$

Therefore, equation (1) has a unique solution in $C(C, \mathbb{R})$ and is Gauss hypergeometric stable.

Proof. Here, we prove that $\Lambda_{k}: Y \longrightarrow Y$ is defined in Theorem 1 with Bielecki's norm $\|.\|_{B}$, which is a contraction operator. Also, we ignore from saying the similar arguments that are expressed in Theorem 1 . Then, for all $\mathscr{J} \in J$, we have

$$
\begin{aligned}
& \left|\Lambda_{k} \rho(\mathscr{J})-\Lambda_{k} w(\mathscr{J})\right| \\
& \leq \frac{1}{\Gamma(\alpha)} \int_{0}^{\mathscr{J}}(\mathscr{J}-\ell)^{\alpha-1}|k(\ell, \rho(\ell), \rho(h(\ell)))-k(\ell, w(\ell), w(h(\ell)))| \mathrm{d} \ell \\
& \leq \frac{\mathbf{L}_{k}}{\Gamma(\alpha)} \int_{0}^{\mathscr{J}}(\mathscr{J}-\ell)^{\alpha-1} \exp (\eta \ell) \\
& \quad\left(\max _{0 \leq \ell \leq q}|\rho(\ell)-w(\ell)| \exp (-\eta \ell)+\max _{0 \leq \ell \leq q}|\rho(h(\ell))-w(h(\ell))| \exp (-\eta \ell)\right) \mathrm{d} \ell \\
& \leq \frac{2 \mathbf{L}_{k}}{\Gamma(\alpha)}\|\rho-w\|_{B} \int_{0}^{\mathscr{J}}(\mathscr{J}-\ell)^{\alpha-1} \exp (\eta \ell) \mathrm{d} \ell .
\end{aligned}
$$
Then,

Now, we utilize Holder's inequality, for $\alpha \in(1 / 2,1)$.

$$
\begin{aligned}
& \int_{0}^{\mathscr{J}}(\mathscr{J}-\ell)^{\alpha-1} \exp (\eta \ell) \leq\left(\int_{0}^{\mathscr{J}}(\mathscr{J}-\ell)^{2(\alpha-1)} \mathrm{d} \ell\right)^{1 / 2}\left(\int_{0}^{\mathscr{J}} \exp (2 \eta \ell) \mathrm{d} l\right)^{1 / 2} \\
& \leq \frac{1}{\sqrt{2 \eta}} \frac{q^{\alpha}}{\sqrt{2 \alpha-1}} \exp (\eta q) .
\end{aligned}
$$




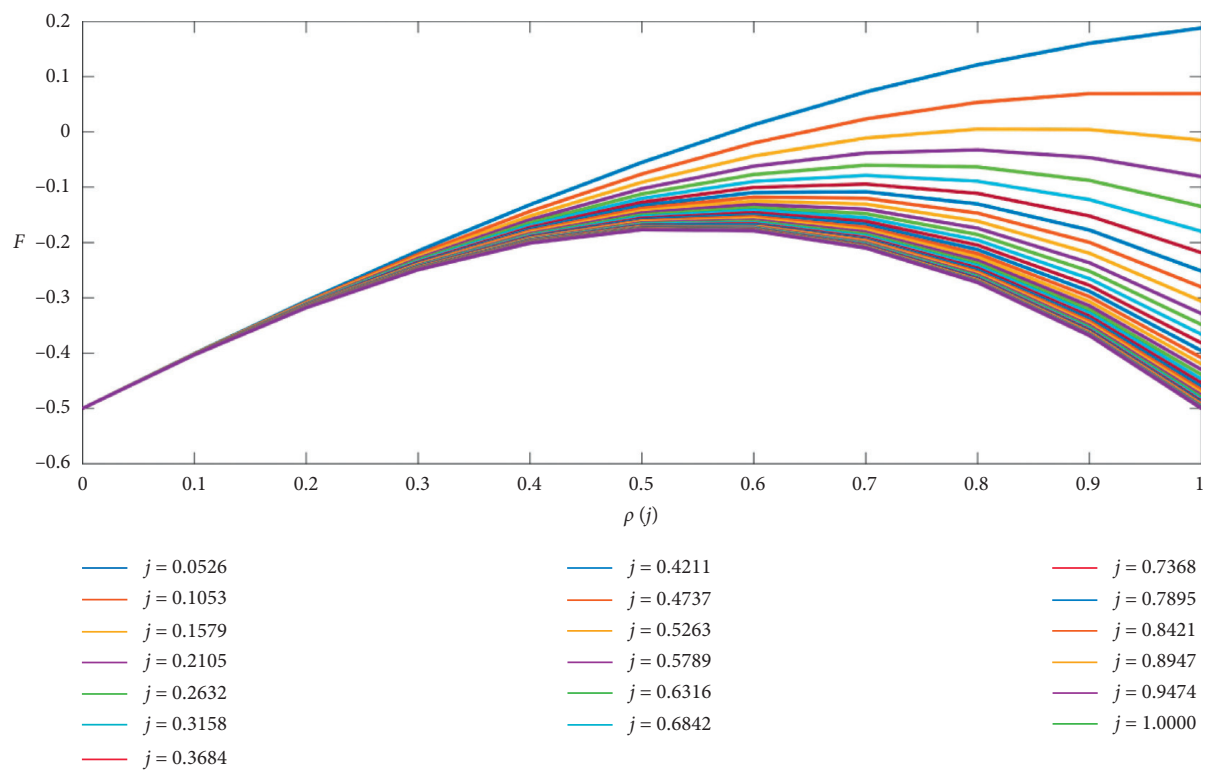

FIGURE 1: Fraction integral equation diagram. $F$ is the nonlinear equation obtained from equation (28).

Therefore,

$$
\left\|\Lambda_{k}(\rho)-\Lambda_{k}(w)\right\|_{B} \leq \frac{2 \mathbf{L}_{k} q^{\alpha} \exp (\eta q)}{\Gamma(\alpha) \sqrt{2(2 \alpha-1) \eta}}\|\rho-w\|_{B} .
$$

Consequently, we have

$$
\left\|\Lambda_{k}(\rho)-\Lambda_{k}(w)\right\|_{B} \leq \frac{2 \mathbf{L}_{k} q^{\alpha} \exp (\eta q)}{\Gamma(\alpha) \sqrt{2(2 \alpha-1) \eta}}\|\rho-w\|_{B}, \quad \rho, w \in C(J, \mathbb{R}) .
$$

Therefore, $\Lambda_{k}$ on $Y$ is a contraction mapping w.r.t. Bielecki's norm $\|.\|_{B}$. Now, we apply the Banach contraction principle to prove (ii). To prove that $T$ in Theorem 1 is a Picard operator, we show that $T$ is a contraction. For all $\mathscr{J} \in J$ and $\alpha \in(1 / 2,1)$ and for every $\sigma, \vartheta \in C\left(J, \mathbb{R}_{+}\right)$, we have

$$
|T \sigma(\mathscr{J})-T \vartheta(\mathscr{J})| \leq \frac{2 \mathbf{L}_{k} q^{\alpha} \exp (\eta q)}{\Gamma(\alpha) \sqrt{2(2 \alpha-1) \eta}}\|\rho-w\|_{B} .
$$

Then,

$$
\|T(\sigma)-T(\vartheta)\|_{B} \leq \frac{2 \mathbf{L}_{k} q^{\alpha} \exp (\eta q)}{\Gamma(\alpha) \sqrt{2(2 \alpha-1) \eta}}\|\rho-w\|_{B} .
$$

Consequently, $T$ is a contraction mapping on $C\left(J, \mathbb{R}_{+}\right)$ with Bielecki's norm $\|.\|_{B}$. The Gauss hypergeometric stability proof is exactly the same as Theorem 1 .

Example 2. Consider a fractional-order differential equation as

$$
{ }^{c} D_{\mathscr{J}}^{2 / 3} \rho(\mathscr{J})=\frac{1}{27} \Gamma\left(\frac{2}{3}\right)(\mathscr{J}-h), \quad \mathscr{J} \in\left(0, \frac{1}{4}\right],
$$

for $\alpha=2 / 3$ and for any $\mathscr{J} \in(0,1 / 4]$. Based on what has been said in Theorem 2 , we have

$$
\rho(\mathscr{J})=w(0)+\frac{1}{27 \Gamma(2 / 3)} \int_{0}^{\mathscr{J}}(\mathscr{J}-\ell)^{-(9 / 10)} \Gamma\left(\frac{2}{3}\right)(\mathscr{J}-0.01) \mathrm{d} \ell .
$$

Let $\mathbf{L}_{k}=1 / 2$ and $\eta=1 / 2$. Consequently,

$$
\frac{2 \mathbf{L}_{k} q^{\alpha} \exp (\eta q)}{\Gamma(\alpha) \sqrt{2(2 \alpha-1) \eta}}=\frac{(1 / 4)^{2 / 3} \exp (1 / 8)}{\Gamma(2 / 3) \sqrt{1 / 3}}<0.58<1 .
$$

According to equation (5) and using the fractional-order differential equation, for the differentiable function $w$, we have

$$
\left|{ }^{c} D_{\mathscr{J}}^{2 / 3} w(\mathscr{J})-k(\mathscr{J}, w(\mathscr{J}), w(\mathscr{J}-h))\right| \leq \varepsilon F\left(\aleph, \beta ; \wp ; \mathscr{J}^{2 / 3}\right) .
$$

All the conditions for the existence of the solution and the Gauss hypergeometric stability are established by Theorem 2. Therefore, the coefficient $c_{F}=F\left(\aleph, \beta ; \wp ;(1 / 4)^{2 / 3}\right)$ exists, and we have

$$
|w(\mathscr{J})-\rho(\mathscr{J})| \leq c_{F} \varepsilon F\left(\aleph, \beta ; \wp ; \mathscr{J}^{2 / 3}\right), \quad \mathscr{J} \in\left(0, \frac{1}{4}\right] .
$$

According to equation (39) for the function $\rho$, we have Figure 2 for every $\mathscr{J} \in(0,1 / 4)$.

Remark 6. In Theorem 2, we examined our problem for $\alpha \in(1 / 2,1)$ by utilizing Holder's inequality. At present, we re-examine the problem for $\alpha \in(0,1)$.

Let ; therefore, we have

$$
\begin{aligned}
\left|\Lambda_{k} \rho(\mathscr{J})-\Lambda_{k} w(\mathscr{J})\right| & \leq \frac{2 \mathbf{L}_{k}}{\Gamma(\alpha)}\|\rho-w\|_{B} \int_{0}^{\mathscr{J}}(\mathscr{J}-\ell)^{\alpha-1} \exp (\eta \ell) \mathrm{d} \ell \\
& \leq \frac{2 \mathbf{L}_{k} q^{\alpha} \exp (\eta q)}{\Gamma(\alpha+1)}\|\rho-w\|_{B} .
\end{aligned}
$$




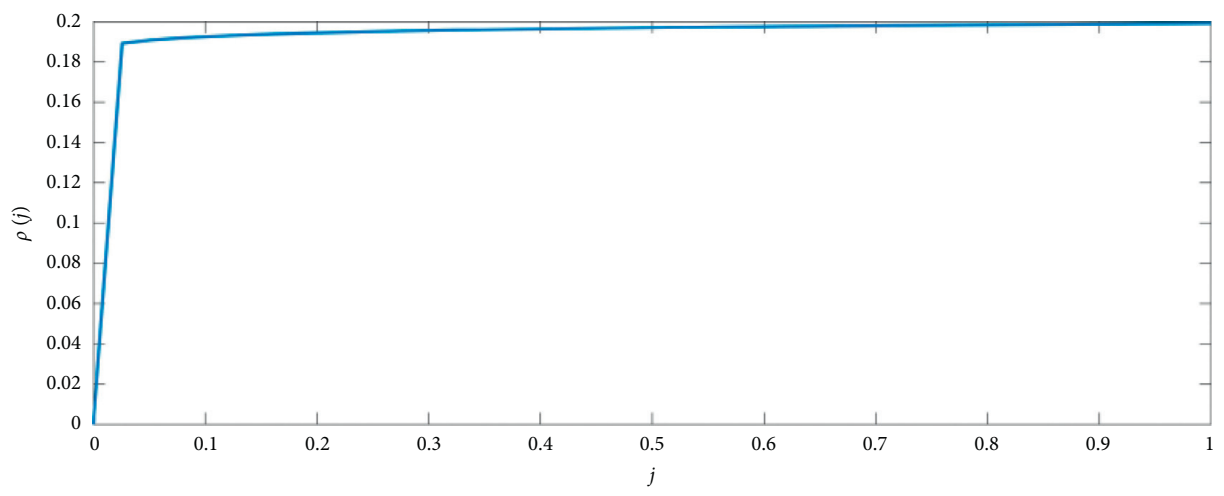

FIGURE 2: Fractional integral equation diagram.

For some $\alpha \in(0,1)$ and $\eta>0$, we have $2 \mathbf{L}_{k} q^{\alpha} \exp (\eta q) / \Gamma(\alpha+1)<1$. Now, we be able demonstrate that $\Lambda_{k}$ is a contraction via Bielecki's norm $\|.\|_{B}$ on $Y$ as well.

\section{Conclusion}

In this paper, we applied the Picard method to investigate existence, uniqueness, and Gauss hypergeometric stability of fractional-order differential equations.

\section{Data Availability}

No data were used to support this study.

\section{Conflicts of Interest}

The authors declare that they have no conflicts of interest.

\section{Authors' Contributions}

All authors participated in its design and coordination, drafted the manuscript, participated in the sequence alignment, and read and approved the final manuscript.

\section{Acknowledgments}

The authors are grateful to the Basque Government for the support of this work under Grant IT1207-19.

\section{References}

[1] F. Liu and K. Burrage, "Novel techniques in parameter estimation for fractional dynamical models arising from biological systems," Computers \& Mathematics with Applications, vol. 62 , no. 3, pp. 822-833, 2011.

[2] R. Hilfer, Applications of Fractional Calculus in Physics, R. Hilfer, Ed., World Scientific Publishing Co., Inc., River Edge, NJ, USA, 2000.

[3] V. Lakshmikantham, S. Leela, and J. Vasundhara Devi, Theory of Fractional Dynamic Systems, CSP, Cambridge, UK, 2009.

[4] S. Longhi, "Fractional Schrodinger equation in optics," Optics Letters, vol. 40, no. 6, pp. 1117-1120, 2015.

[5] A. Liemert and A. Kienle, "Fractional Schrödinger equation in the presence of the linear potential," Mathematics, vol. 4, no. 2, pp. 31-45, 2016.
[6] A. R. El-Nabulsi, "Fractional derivatives generalization of Einstein's field equations," Indian Journal of Physics, vol. 87, no. 2, pp. 195-200, 2013.

[7] Qualitative Analysis of Multi-Terms Fractional Order Delay Differential Equations via the Topological Degree Theory.

[8] M. Sher, K. Shah, M. Feckan, and R. Khan, "Qualitative analysis of multi-terms fractional order delay differential equations via the topological degree theory," Mathematics, vol. 8 , no. 2 , p. $218,2020$.

[9] W. Li, J. Ji, and L. Huang, "Dynamics of a controlled discontinuous computer worm system," Proceedings of the American Mathematical Society, vol. 148, no. 10, pp. 43894403, 2020.

[10] W. Li, J. Ji, L. Huang, and Z. Guo, "Global dynamics of a controlled discontinuous diffusive SIR epidemic system," Applied Mathematics Letters, vol. 121, Article ID 107420, 2021.

[11] G. Xiao and J. Wang, "Representation of solutions of linear conformable delay differential equations," Applied Mathematics Letters, vol. 117, no. 6, Article ID 107088, 2021.

[12] L. Suo, M. Fečkan, and J. Wang, "Quaternion-valued linear impulsive differential equations," Qualitative Theory of Dynamical Systems, vol. 20, no. 2, p. 33, 2021.

[13] J. Wang and Y. Zhang, "Ulam-Hyers-Mittag-Leffler stability of fractional-order delay differential equations," Optimization, vol. 63, no. 8, pp. 1181-1190, 2014.

[14] A. A. Kilbas, H. M Srivastava, and J. J. Trujillo, "Theory and applications of fractional differential equations," North-Holland mathematics studies, Vol. 204, Elsevier, Amsterdam, Netherlands, 2006.

[15] I. A. Rus, "Ulam stability of ordinary differential equations," Studia Universitatis Babes-Bolyai Mathematica, vol. 54, pp. 125-133, 2009.

[16] J. R. Wang and X. Li, " $E_{\alpha}$-Ulam type stability of fractional order ordinary differential equations," Journal of Applied Mathematics and Computing, vol. 45, no. 1-2, pp. 449-459, 2014.

[17] S. András and A. R. Mészáros, "Ulam-Hyers stability of dynamic equations on time scales via Picard operators," Applied Mathematics and Computation, vol. 219, no. 9, pp. 4853-4864, 2013.

[18] I. A. Rus, "Problem 6.7 Open problems in control of linear discrete multidimensional systems," Unsolved Problems in Mathematical Systems and Control Theory, vol. 70, pp. 221228, 2009.

[19] K. Liu, M. Fečkan, and J. Wang, "Hyers-Ulam stability and existence of solutions to the generalized liouville-caputo fractional differential equations," Symmetry, vol. 12, no. 6, p. 955, 2020. 
[20] M. Abramowitz and I. A. Stegun, Handbook of Mathematical Functions with Formulas, Graphs, and Mathematical Tables, M. Abramowitz and I. A. Stegun, Eds., Dover Publications, Inc., New York, NY, USA, 1992.

[21] D. H. Hyers, G. Isac, and T. M. Rassias, "Stability of functional equations in several variables," Progress in Nonlinear Differential Equations their Applications, Birkhäuser, vol. 34, Boston, MA, USA, 1998.

[22] S. Rezaei Aderyani, R. Saadati, and D. O’Regan, "Existence, uniqueness and Ulam-Hyers-Mittag-Leffler stability of a class of $\Delta$-Hilfer fractional differential equations via the CădariuRadu method," Preprint, 2021.

[23] I. Ahmad, K. Shah, G. Rahman, D. Baleanu, and B. Dumitru, "Stability analysis for a nonlinear coupled system of fractional hybrid delay differential equations," Mathematical Methods in the Applied Sciences, vol. 43, no. 15, pp. 8669-8682, 2020.

[24] G. Choi, S.-M. Jung, and J. Roh, "An operator method for the stability of inhomogeneous wave equations," Symmetry, vol. 11 , no. 3, p. 324, 2019. 